\title{
Detailed simulations of particle breakage as a result of agitation
}

\author{
Jinghan Xie ${ }^{\mathrm{a}}$, Lijuan Zhang ${ }^{\mathrm{a}}$, J.J. Derksen ${ }^{\mathrm{b}}$
}

${ }^{a}$ School of Chemistry and Chemical Engineering, Shanghai University of Engineering Science, Shanghai 201620, China

${ }^{\mathrm{b}}$ School of Engineering, University of Aberdeen, Aberdeen, UK

*jderksen@abdn.ac.uk

Submitted to Chemical Engineering Research \& Design - November 2020

Revision submitted: February 2021

Accepted: February 2021

\begin{abstract}
Two-way coupled simulations of the solids-liquid flow in a lab-scale mixing tank operating at a Reynolds number of 4,000 and a solids volume fraction of $10 \%$ have been performed. The simulations keep track of individual particles, including their collisions. Upon a collision a particle is broken if the magnitude of the momentum exchanged in the collision exceeds a threshold value. The latter value is a measure for the strength of the particle. We observe how the flow systems, with ever decreasing particle sizes, evolve in time and quantify which collisions at what location primarily lead to breakage. The revolving impeller is the major source of breakage, either directly through particle-impeller collisions or by high-impact particleparticle collisions in the impeller-swept volume.
\end{abstract}

\section{Keywords}

Solids-liquid flow, mixing, particle breakage, computational fluid dynamics 


\section{Introduction}

Manufacturing of particulate materials through precipitation in a supersaturated liquid solution is common in the process and pharmaceutical industries. For achieving high levels of heat and / or mass transfer, fluid flow, often turbulent, is an essential part of such crystallization operations. From the perspective of the crystals, the interaction between solids and liquid has impact on their growth or dissolution, their aggregation and their breakage (of primary particles or aggregates).

This paper focuses on particle breakage and does so by means of numerical simulations. Breakage in agitated solids-liquid suspensions has been studied extensively with important papers on experimental work by Mersmann and co-workers [1-3] and Asakuma et al [4]. They identified collisions of crystals as the predominant cause of their breakage. The intensity of collisions experienced by particles moving through a liquid phase depends upon fluid, particle, and flow properties. The mechanical strength of a particle determines if it breaks upon colliding with a certain intensity. For non-colloidal particles, i.e. particles large enough not to be influenced by Brownian motion, collisions are largely driven by inertia as well as fluid velocity gradients. The key dimensionless quantity in this respect is the Stokes number $\mathrm{Stk}=\tau_{p} / \tau_{f}$ with $\tau_{p}$ the (inertial) relaxation time of the particle and $\tau_{f}$ a dominant flow time scale. In solids-liquid flow systems with Stokes numbers $\mathrm{Stk} \geq 1$ collisions occur due to particles diverging from streamlines and so encountering other particles [5]. If Stk $\ll 1$ particles follow the flow and velocity gradients on length scales comparable to particle size are required for collisions [6]. For Stk $\gg 1$, collisions are mostly determined by the dynamics of the solids, less so by the fluid dynamics [7]. However, different from solids-gas systems, agitated solids-liquid systems do not reach very high Stokes numbers.

It is thus clear that particle behavior in liquids - and specifically particle collisions and breakage - are intimately related to flow. For that reason, computational fluid dynamics studies involving the behavior of solid particles in liquid have been undertaken to complement and guide experimental work [8-11]. Including breakage phenomena in such simulations often goes through population balance modeling. The evolution of the particulate system in physical space as well as property space (which most often involves particle size) is then described by a population balance equation (PBE) $[12,13]$. Changes in particle size are modeled in the 
PBE with "kernel functions". These function contain the physics of flow and solids behavior responsible for particles changing size, not necessarily only as a result of breakage but also due to e.g. growth/dissolution and aggregation [13]. The quality of results generated through a PBE approach are thus highly depended on the ability of the kernels to capture the essential physics and the accuracy of model parameters that often are derived from experimental results.

In this paper we try out a different approach of keeping track of the evolution of an agitated particulate system with breakage. Instead of solving a population balance, we monitor the motion of individual particles, including their collisions. The intensity of a collision (more precisely defined below) determines if it results in breakage of a particle. An advantageous aspect of this approach is that collisions are dealt with from first, classical mechanical, principles and do not need to be modeled based on statistical (local) flow and particle properties. A disadvantage is that for computational reasons we need to restrict ourselves to small systems while having an appreciable solids volume fraction. This is mainly to limit the number of particles to be tracked in a simulation. We want to study high solids loading suspension given their relevance in industry and to have systems for which particle-particle collision are an important factor in their overall behavior.

The aim of this paper is (1) to present our numerical approach and apply it in a small-scale agitated tank, (2) to quantify the types of collisions (among particles, with the impeller, with the container wall) that most likely lead to breakage, and (3) to study how the evolving particulate system with particles gradually getting smaller in size feeds back to the behavior of the two-phase system as a whole. At this stage of our research we have made the strong assumption that if particles break that they break in two equal parts. This is not essential for our methodology. However, it does help us limiting the parameter space to be studied.

This paper has been organized in the following manner. The next section characterizes the flow system: geometry, (dimensionless) parameters characterizing the flow and particulate system, including breakage criteria. We then describe the numerical methodology with an emphasis on breakage modeling. Physical and numerical settings of the simulations are discussed next. The results comprise of qualitative impressions of the two-phase flow and the way the system evolves in time, including its particle size 
distribution for a range of particle strengths. The final section provides conclusions and an outlook to future work.

\section{Flow system characteristics}

The lab-scale flow system we consider is a rectangular mixing tank with a square footprint with side-length $L$, liquid height $H=L$, and volume $V=L^{2} H$ see Figure 1 that also defines the Cartesian coordinate system to be used throughout the paper. The contents of the tank are stirred by an impeller with diameter $D$. The impeller consists of four flat blades, placed under an angle of $45^{\circ}$ and mounted on a hub. This so-called pitched-blade turbine is set to rotate with a rotational velocity $N$ (revolutions per unit time) in such a way that it pumps the liquid in a downward direction (the negative $z$-direction). The tank contains a Newtonian liquid and spherical solid particles. The liquid density is $\rho$, its kinematic viscosity is $\nu$. Initially all $n_{0}$ particles have the same diameter $d_{0}$. The material the particles are made of has density $\rho_{s}>\rho$. The overall solids volume fraction in the tank then equals $\langle\phi\rangle=\frac{n_{0} \pi d_{0}^{3}}{6 \mathrm{~V}}$. The Reynolds number associated with the impeller motion is defined as $\operatorname{Re}=N D^{2} / \nu$.

The particles feel a net gravity force in the negative $z$-direction. The competition between gravity pulling the particles to the bottom and the flow generated by the spinning impeller suspending the particles is quantified through the Shields number which here is defined as $\theta=\frac{\rho N^{2} D^{2}}{g \Delta \rho d_{0}}$ with $g$ gravitational acceleration and $\Delta \rho=\rho_{s}-\rho$. With the Shields number in this form it is assumed that inertial stress (that scales with $\rho N^{2} D^{2}$ ) is more relevant than viscous stress for suspending the particles.

As stated in the introduction, the main aim of this paper is to assess how the two-phase flow dynamics promotes breakage of the solid particles. It is assumed that collisions are the only reason for breakage of particles. We consider three types of collisions that can cause breakage: with another particle, with the impeller and with the container walls. The main independent variable in this study is the strength of the particulate material. That strength has been defined and quantified in terms of a speed. It is the minimum speed of impact $u_{c}$ of a particle of diameter $d_{0}$ with a rigid solid wall that would lead to breakage. The 
advantage of this definition is that it allows for easy experimentation. If the particle is dropped (in vacuum) on a horizontal solid wall, $u_{c}=\sqrt{2 g h_{c}}$ with $h_{c}$ the smallest height that results in breakage. In the next section - on computational methods - we will show how this definition is used in the simulations to assess if a collision leads to breakage of a particle. Simulation results will be presented in non-dimensional terms where the minimum speed of impact has been scaled with the tip speed of the impeller $\mathrm{v}_{t i p}=\pi N D$.

\section{Numerical methods}

\section{Fluid flow \& particle dynamics}

The liquid flow has been solved by the lattice-Boltzmann (LB) method $[14,15]$. For this the tank volume has been divided in cubic cells that all have the same size. In each cell the LB method keeps track of a discretized version of the velocity distribution function. Local fluid velocity and pressure are derived from summations over all velocity directions [14]. In order to account for the presence of solid particles, a variant of the LB method has been used that solves the volume-averaged continuity and Navier-Stokes equations [16]

$$
\begin{gathered}
\frac{\partial}{\partial t}\left(\rho \phi^{c}\right)+\nabla \cdot\left(\rho \phi^{c} \mathbf{u}\right)=0 \\
\frac{\partial}{\partial t}\left(\rho \phi^{c} \mathbf{u}\right)+\nabla \cdot\left(\rho \phi^{c} \mathbf{u u}\right)=\phi^{c} \nabla \cdot \boldsymbol{\pi}+\mathbf{f}_{\mathrm{s}}
\end{gathered}
$$

where $\mathbf{u}$ is the interstitial liquid velocity, $\phi^{c}=1-\phi$ the local liquid volume fraction (and $\phi$ the solids volume fraction), $\boldsymbol{\pi}$ the stress tensor, and $\mathbf{f}_{\mathrm{s}}$ the force per unit volume exerted by the solid particles on the fluid. Since at every moment in time the location of the particles is known, $\phi$ and thus $\phi^{c}$ can be determined by mapping the particles on the Eulerian (LB) grid [16]. Mapping of Lagrangian (particle-related) information on the Eulerian grid as well as mapping Eulerian information on the particles makes use of a mapping function for which we use a clipped fourth-order polynomial [17]. It has a near-Gaussian shape and has the advantage over a Gaussian that it is computationally more efficient and is zero outside its (finite) width. In earlier work [18] we shown favorable results if the half-width of the mapping function is equal to 
1.5 times the particle diameter. Therefore the width of the mapping function of a particle is a function of its size.

The dynamics of the particles is accounted for by solving there linear equations of motion with the particles feeling gravity, drag and contact forces. The drag force model takes into account the particle-based Reynolds number as well as the local solids volume fraction: $\mathbf{F}_{\mathbf{D}}=3 \pi \rho v d\left(\mathbf{u}-\mathbf{u}_{\mathbf{p}}\right) F\left(\operatorname{Re}_{p}, \phi\right)$ with $\mathbf{u}_{\mathbf{p}}$ the velocity of the particle and $d$ its diameter. The function $F$ is written as $F\left(\operatorname{Re}_{p}, \phi\right)=p\left(\operatorname{Re}_{p}\right) q(\phi)$. Here $p\left(\operatorname{Re}_{p}\right)=\left(1+0.15 \operatorname{Re}_{p}^{0.687}\right)$ is the Schiller-Naumann [19] drag correlation with $\operatorname{Re}_{p}=(1-\phi)\left|\mathbf{u}-\mathbf{u}_{\mathbf{p}}\right| d / v$, and $q(\phi)=(1-\phi)^{-2.65}$ Wen \& Yu [20] drag.

The contact force is assumed to be radial. For a particle-particle contact this implies that it works on the line connecting the centers of the particles. For a particle-impeller or particle-wall contact the contact force is in the direction normal to the impeller or wall surface. The contact force has a repulsive linear elastic part that is proportional to the overlap distance and a lubrication part that is proportional to the relative velocity [21]. The lubrication force gets activated before particles touch or overlap; it represents the effect of the liquid film being squeezed out between two approaching solid surfaces (repulsive force) or sucked in between separating surfaces (attractive force). The presence of a lubrication force does not imply inelasticity in the actual "dry" collision. Settings of contact force parameters are the same as in [16].

\section{Breakage modeling}

A collision between two particles $i$ and $j$ having mass $m_{i}$ and $m_{j}$ respectively is described as $\mathbf{u}_{\mathbf{p}, \mathbf{i}}^{*}=\mathbf{u}_{\mathbf{p}, \mathbf{i}}+\mathbf{J} / m_{i}$ and $\mathbf{u}_{\mathbf{p}, \mathbf{j}}^{*}=\mathbf{u}_{\mathbf{p}, \mathbf{j}}-\mathbf{J} / m_{j}$. The $*$ indicates post-collision velocities. The momentum exchange vector $\mathbf{J}$ generally has a normal and tangential component with in the majority of collisions the normal (radial) component significantly larger than the tangential components [22]. We therefore only consider the radial component of $\mathbf{J}$, i.e. we assume $\mathbf{J}=J_{n} \mathbf{n}$ with $\mathbf{n}$ the unit vector pointing from particle $i$ to $j$ and [22]

$$
J_{n}=\frac{(1+e) \mathbf{n} \cdot\left(\mathbf{u}_{\mathbf{p}, \mathbf{j}}-\mathbf{u}_{\mathbf{p}, \mathbf{i}}\right)}{1 / m_{i}+1 / m_{j}} .
$$


The restitution coefficient has been set to $e=1$ (dry collisions are assumed to be elastic). Note that $J_{n}<0$ since only two approaching particles do collide. We assume that breakage occurs if $-J_{n}$ exceeds a critical value that we derive from the critical velocity $u_{c}$ introduced above. For a particle with diameter $d_{0}$ and mass $m_{0}=\frac{\pi}{6} \rho_{s} d_{0}^{3}$ falling on a horizontal wall with velocity $u_{c}$ the normal momentum exchange is $\left|J_{n}\right|=2 m_{0} u_{c}$ so that $2 m_{0} u_{c}$ is the critical momentum exchange value for breakage.

In the simulation we determine for every detected collision the normal momentum exchange according to Eq. 3 (with $e=1$ ). If the collision is between particle $i$ and the tank wall then $m_{j} \rightarrow \infty$ and $\mathbf{u}_{\mathbf{p}, \mathbf{j}}=\mathbf{0}$. If the collision is between particle $i$ and the impeller then $m_{j} \rightarrow \infty$ and $\mathbf{u}_{\mathbf{p}, \mathbf{j}}=\mathbf{\Omega} \times \mathbf{r}$ with the angular velocity vector of the impeller $\boldsymbol{\Omega}=2 \pi N \mathbf{e}_{\mathrm{z}}$ and $\mathbf{r}$ the location of the collision.

Once a breakage event has been identified the following assumptions apply: (1) a particle with diameter $d_{i}$ breaks into two equal fragments with diameter $d_{i} / \sqrt[3]{2} ;(2)$ if two particles of unequal size collide only the larger particle breaks; (3) if two particles of equal size collide only one of them - randomly picked - breaks. From (1) it is clear that we assume the breakage fragments are spherical - which admittedly is a quite a strong assumption.

It is important, specifically in flow regions with high solids volume fraction, to carefully place the fragments in order to avoid as much as possible large overlap with surrounding particles. Figure 2 illustrates how this placement has been done.

\section{Simulation settings}

The base-case system has a Reynolds number of $\operatorname{Re}=4,000$, a Shields number of $\theta=260$, and an overall solids volume fraction of $\langle\phi\rangle=0.098$. The initial diameter of the particles relative to the impeller diameter is $d_{0} / D=0.021$ and the initial number of particles is 250,000 . The density ratio is $\rho_{s} / \rho=2.23$. The number of grid cells in each coordinate direction is 110 . The impeller makes one revolution in 1600 time steps. The initial particle size $d_{0}$ is equal to the grid spacing. In [16] we have shown that the numerical 
procedure is well capable of dealing with particles of sizes comparable to or even larger than the grid spacing by demonstrating grid convergence.

Although the base-case has been fully defined in terms of dimensionless numbers, it might help the interpretation of the results if we also describe it in physical units. Then the volume of the tank is $110 \times 110 \times 110 \mathrm{~mm}^{3}$ (1.33 liter); the impeller has a diameter of $D=48 \mathrm{~mm}$, and the initial particle diameter is $d_{0}=1 \mathrm{~mm}$. The impeller makes $37 \mathrm{rev} / \mathrm{s}(\sim 2000 \mathrm{rpm})$. The density of the liquid is $1000 \mathrm{~kg} / \mathrm{m}^{3}$ and its kinematic viscosity is $\nu=2.1 \cdot 10^{-5} \mathrm{~m}^{2} / \mathrm{s}$ ( $\sim 20$ times water). Gravitational acceleration is $g=9.8 \mathrm{~m} / \mathrm{s}^{2}$.

The strength of the particles is the main independent variable. It has been varied such that the critical velocity divided by the tip speed of the impeller is in the range $0.1 \leq u_{c} / \mathrm{v}_{t i p} \leq 0.5$. In the physical base-case $\mathrm{v}_{\text {tip }}=5.6 \mathrm{~m} / \mathrm{s}$ so that $u_{c}$ would be in the range of 0.56 to $2.8 \mathrm{~m} / \mathrm{s}$.

At the start of a simulations, the particles (all of diameter $d_{0}$ ) are at rest and form a loosely packed bed of thickness $16 d_{0}$ on the bottom of the tank. At that moment also the liquid has zero velocity. At time $t=0$ we start the impeller and slowly increase its angular velocity until it reaches its final, steady value $N$. This start-up phase is such that over its duration of $2 / N$ the impeller completes one revolution. The simulations run over at least 200 impeller revolutions which is - in the physical system - a duration of only $5.4 \mathrm{~s}$.

Given that the simulation is started with all particles of the same size and that if particles break they break in two equal parts, there only is a small number of particle diameters in a simulation. In most simulations we keep track of 6 particle diameters. This means that a particle can break up to 5 times and acquire a volume of $2^{-5} \frac{\pi}{6} d_{0}^{3}$. The breakage criterion makes it increasingly hard to break smaller particles given the way $J_{n}$ depends on the mass of the particles (Eq. 3) and the rule that in a collision between two unequal particles only the larger one breaks.

\section{Results}

\section{Impressions}

In Figure 3 we show a time sequence of one of the simulations - the base case with particle strength so that $u_{c} / \mathrm{v}_{\text {tip }}=0.2$. It shows particle locations with the particles colored according to their size, as well as 
instantaneous liquid velocity magnitude contours in a vertical plane through the center of the tank. In order to be able to look into the two-phase mixing system, in the later stages of the suspension process (from $t N=10$ onward) only particles present in a mid-slice are shown in the figure. We see the suspension process starting up as a result of the mostly downward stream of liquid coming off the impeller penetrating the particle bed. Already in the initial stages $(t N \leq 5)$, breakage occurs as witnessed form the appearance of red particles. The flow generated by the impeller does not reach the region directly underneath the hub (the cylinder on which the blades are mounted) very well leading to a relatively stagnant zone there from which particles hardly get suspended. This zone persist for at least 50 impeller revolutions.

Over time, breakage shifts the particle size distribution to smaller sizes. As can be seen, this leads to a more homogeneous distribution of particles over the tank volume. If one compares the snapshots at 50 and 200 impeller revolution one sees that regions void of particles (the "holes" in the particles slices in Figure 3) have become substantially smaller at the later moment. These holes are due to larger particles avoiding highvorticity regions and preferentially concentrating at the edges of vortices. This effect scales with the Stokes number [23]. A meaningful estimate of the Stokes number is based on the flow time scale set equal to the reciprocal of the blade passage frequency. With four blades: $\tau_{f}=1 /(4 N)$. The particle relaxation time is $\tau_{p}=\frac{\rho_{p}}{\rho} \frac{d^{2}}{18 \nu}$ so that $\operatorname{Stk}=\frac{\rho_{p}}{\rho} \frac{2 N d^{2}}{9 \nu}$. The initial Stokes number $\left(\right.$ with $\left.d=d_{0}\right)$ is $\operatorname{Stk}_{0}=0.85$. As we will see when we discuss how the Sauter mean diameter evolves in time, after 200 impeller revolutions this has reduced to $\mathrm{Stk} \approx 0.45$.

Figure 4 gives a different perspective of the particle suspension process. It shows the short-term averaged solids volume fraction - indiscriminate of particle size - in a vertical cross section at four moments in time. The first frame at $t N=10$ is in the start-up phase with still no solids in large regions in the top of the tank. Then - at $t N=50$ - we see the solids "cone" underneath the hub in the center of the tank. The particle size reduction due to breakage and the easier suspension of the smaller particles contributes to the disappearance of the cone at later times. Eventually the system gets well suspended with in the bulk a fairly homogeneous solids volume fraction and some preferential concentration at the walls. 
A more detailed view of particles in the direct vicinity of the impeller at $t N=100$ is given in Figure 5. It shows the presence of particles of sizes down to $d=2^{-4 / 3} d_{0}$ (black). The smallest size particles in the simulation with $d=2^{-5 / 3} d_{0}$ are not present in Figures 3 and 5. A minute number of them does get generated in the simulations and will be discussed when particle size distributions are being presented.

\section{Temporal evolution}

We are dealing with an inherently unsteady two-phase flow system because of the gradual decline in particle sizes due to breakage. We ultimately would reach a steady state when particles have become so small that none of them breaks anymore. In this paper, however, we are primarily interested in transient behavior. Figure 6 shows the evolution of the total number of particles as well as their average size, the latter in the form of the Sauter mean diameter $d_{32}$, over a time span of 200 impeller revolutions. Over this time, the number of particles increases by a factor of more than 5 for the weaker particles with strength $u_{c} / \mathrm{v}_{\text {tip }}=0.1$, and by a factor of two if $u_{c} / \mathrm{v}_{t i p}=0.4$. The time series of $d_{32} / d_{0}$ shows that breakage obviously results in the particles on average getting smaller to an extent that is a function of their strength.

The way breakage is distributed over the three types of collisions (particle-particle, particle-impeller and particle-wall) strongly depends on particle strength as well as on the moment in time, see Figure 7. In all cases shown, particle-wall collisions contribute only marginally to the breakage frequency. Breakage requires a minimum relative velocity between the two elements taking part in a collision. With low liquid velocities in near wall regions and Stokes numbers that do not exceed unity, the chances of a particle hitting the wall with a speed sufficiently high for breakage are small.

For the weakest particles (with $u_{c} / \mathrm{v}_{\text {tip }}=0.1$ ) the contribution of particle-particle and particle-impeller collisions to the total number of breakage events are of a comparable magnitude. Stronger particles, however, are mostly broken by the impeller. For $u_{c} / \mathrm{v}_{t i p}=0.4$ more than $90 \%$ of the breakage in the time period of 200 revolutions is directly due to the impeller. The dominance of the impeller in promoting breakage is even more pronounced when we study the location of the breakage events, see Figure 8 . The 
color contours in Figure 8 represent $x=\frac{n_{b v}}{n_{0} / V}$. This means that the number of particles broken per unit volume at a certain location per impeller revolution is $x$ times the - on average - number of particles per unit volume present at that location.

Breakage due to particle-particles collisions is almost exclusively confined to the volume swept by the impeller. Particles having gained high velocities by the action of the impeller, either due to direct contact with the impeller or due to transmission via the liquid flow, are able to break other particles in their direct surroundings. Some breaking of particles by other particles outside the impeller swept volume takes place in the impeller outstream, relatively close to the impeller.

After reaching peak values at $t N \approx 20$ to 30 , the total numbers of breakage events per impeller revolution decays in time, see Figure 7 . This has two reasons. In the first place because smaller particles need higher relative velocities to be broken, see Eq. 3. In the second place because smaller particles more closely follow the liquid flow leading to a reduction of relative velocities among particles and between particles and the impeller blades and thus to a reduction of impactful collisions.

Figure 8 also shows the locations on the impeller where particles preferentially break. These are the top edges and the inner corners where the blades are attached to the hub. The top edges are the leading edges of the blade and in the "front line" for encounters between impeller blades and particles. The high breakage frequencies at the inner corners are a less obvious phenomenon. It likely is the result of a small vortex in front of the blades at the corner location, see Figure 8. The vortex creates a dead zone that traps particles, i.e. particles there are not carried away by the high speed flow of liquid around the impeller blades. These trapped particles have a significant chance of being hit and subsequently broken by the impeller.

A set of overall, time-averaged liquid flow characteristics in the vertical center plane are shown in Figure 10. The three quantities depicted there (velocity magnitude $|\mathbf{u}|$, kinetic energy contained in the velocity fluctuations - periodic plus random $-k$, and the energy dissipation rate $\varepsilon$ ) all point to a very inhomogeneous flow with strong activity in the impeller swept volume and in the impeller outstream and mild conditions elsewhere. As we have seen, particle breakage is confined to these high activity regions and 
virtually absent elsewhere in the mixing tank. From a practical perspective, impeller design is crucial in controlling (enhancing or avoiding) particle breakage.

\section{Size distribution}

Particle size distributions are shown in Figure 11. We distinguish between 6 particle classes $q=0 \ldots 5$ with $q=0$ the initial particles with diameter $d_{0}$ and the subsequent classes such that the particle volume is half that of the volume in the previous class.

The shape of the size distributions strongly depends on the particle strength. Where for $u_{c} / \mathrm{v}_{\text {tip }}=0.1$ the initial particles almost all have been broken after 200 impeller revolution, the initial particles still make up $40 \%$ of the total particle volume for $u_{c} / \mathrm{v}_{t i p}=0.4$.

Six particle classes is sufficient for the flow system we have been simulating given that for none of the particle size distributions shown in Figure 10 the smallest class $(q=5)$ represents an appreciable solids volume. As noted above, in most cases breakage due to collisions with the impeller is the dominant mechanism. For such collisions (given Eq. 3 with one of the masses - the impeller - going to infinity) $J_{n}=2 m_{i} \mathbf{n} \cdot\left(\mathbf{u}_{\mathbf{i m p}}-\mathbf{u}_{\mathbf{p}, \mathbf{i}}\right)=2 m_{i} u_{r n}$ with $u_{r n}$ the normal relative velocity of particle and impeller. Particles break if $\left|J_{n}\right| \geq 2 m_{0} u_{c}$. For a particle in size class $q$ to be broken by the impeller we thus need a normal relative velocity of $\left|u_{r n}\right|>2^{q} u_{c}$. It thus is unlikely that even the weakest particles in this study - those with $u_{c} / \mathrm{v}_{\text {tip }}=0.1$ - would break to class $q=5$ particles as it would require a relative velocity of at least $\left|u_{r n}\right|=2{ }^{4} u_{c}=1.6 \mathrm{v}_{t i p}$.

\section{Summary, conclusions \& outlook}

The paper presents results of simulations of solids-liquid flow in a lab-scale agitated tank with the solid particles breaking as a result of collisions. The main purpose of the paper is to quantify the relative importance of breakage mechanisms in relation to the mechanical strength of the particles. The focus is on systems with relatively high solids loadings (solids volume fraction of $\sim 10 \%$ ) with frequent collisions between particles and between particles and the revolving impeller. 
In most cases investigated, the impeller is the major cause of breakage, either directly when a particles hits an impeller blade, or indirectly through breakage as a result of two colliding particles in the impeller swept volume. Only for very low-strength particles breakage through particle-particle collisions contributes appreciably to breakage.

The simulations clearly show the impact of particle size reduction due to breakage on the two-phase flow characteristics. As a result of the Stokes number falling well below one in the course of the mixing process the homogeneity of the spatial distribution of particles increases as does their level of suspension in the liquid flow.

From a practical perspective there are clear limitations to the simulations presented here that require further work to be overcome. The assumption that particles break in two parts of equal volume is a major simplification. It will be interesting to study the sensitivity of this assumption by including a probabilistic model that captures the statistics of the fragment size distribution upon breakage [1], or - better - to involve experimental data on fragment size distributions of particles that break in a collision. We do not feel that the assumption that breakage products are considered spherical particles is a major simplification. This in light of other approximations such as the levels of spatial resolution of the liquid flow. The shape of the particles is mainly represented by a drag coefficient, i.e. the liquid flow is not resolved such as to capture the shape of the particles.

Our results mainly apply to a lab-scale tank given the relatively low (transitional) Reynolds number $(\operatorname{Re}=4,000)$ and the particle being large relative to tank size. This needs to be extended to more practically relevant scales but - at the same time - could be helpful when devising small scale experiments for validation purposes. These are not only essential for validation but also for guiding the simulation research by identifying sensitivities and physical effects of key importance that the simulations should focus on. 


\section{References}

[1] A. Chianese, R. Sangl, A. Mersmann, On the size distribution of fragments generated by crystal collisions, Chem. Eng. Commun. 146 (1996) 1-12.

[2] C. Gahn, A. Mersmann, The brittleness of substances crystallized in industrial processes. Powder Technol. 85 (1995) 71-81.

[3] C. Gahn, A. Mersmann, Theoretical prediction and experimental determination of attrition rates, Trans. Instn. Chem. Eng. 75A (1997) 125-131.

[4] Y. Asakuma, T. Honda, K. Maeda, H. Miki, K. Fukui, Fragmentation behavior of aggregated crystal in suspension crystallization processes. Powder Technol. 181 (2008) 266-272.

[5] A. Ten Cate, J.J. Derksen, L.M. Portela, H.E.A. Van den Akker, Fully resolved simulations of colliding spheres in forced isotropic turbulence, J Fluid Mech. 519 (2004) 233-271.

[6] M. Von Smoluchowski, Versuch einer mathematischen theorie der Koagulationskinetik Koloider Loesungen, Z. Phys. Chem. 92 (1917) 156.

[7] Z. Peng, Y.A. Alghamdi, B. Moghtaderi, E. Doroodchi, CFD-DEM investigation of transition from segregation to mixing of binary solids in gas fluidised beds, Advanced Powder Technology 27 (2016) $2342-2353$.

[8] J.J. Derksen, Numerical simulation of solids suspension in a stirred tank, AIChE J. 49 (2003) 27002714.

[9] G. Montante, F. Magelli, Modeling of solids distribution in stirred tanks: analysis of simulation strategies and comparison with experimental data, Int. J. Comput. Fluid Dyn. 19 (2005) 253-262.

[10] A. Tamburini, A. Cipollina, G. Micale, A. Brucato, M. Ciofalo, CFD simulations of dense solid-liquid suspensions in baffled stirred tanks: Prediction of suspension curves, Chem. Eng. J. 178 (2011) 324341.

[11]X. Wu, T. Li, J. Cai, Z. Peng, Z. Yuan, Numerical prediction of particle number concentration distribution in scrubbing-cooling chamber of entrained-flow coal gasifier, Chemical Engineering Journal 149 (2009) 325-333.

[12] D. Ramkrishna, Population balances - Theory and applications to particulate systems in engineering, Academic Press, London, 2000.

[13] A. Mersmann, B. Braun, M. Loffelmann, Prediction of crystallization coefficients of the population balance. Chem. Eng. Sc. 57 (2002) 4267-4275.

[14] S. Succi, The lattice Boltzmann equation for fluid dynamics and beyond, Clarendon Press, Oxford, 2001.

[15] S. Chen, G.D. Doolen, Lattice Boltzmann method for fluid flows. Annu. Rev. Fluid Mech. 30 (1989) $329-364$. 
[16] J.J. Derksen, Eulerian-Lagrangian simulations of settlingand agitated dense solid-liquid suspensionsachieving grid convergence, AIChE J. 64 (2018) 1147-1158.

[17] N.G. Deen, M. Van Sint Annaland, J. Kuipers, Multi-scale modeling of dispersed gas-liquid two-phase flow. Chem. Eng. Sc. 59 (2004) 1853-1861.

[18] J.J. Derksen, Assessing Eulerian-Lagrangian simulations of dense solid-liquid suspensions settling under gravity, Comput. Fluids 176 (2018) 266-275.

[19] L. Schiller, A. Naumann, Uber die grundlagen den Berechnungen bei der Schwerkraftaufbereitung, Ver. Deut.Ing. Z. 77 (1933) 318-320.

[20] C.Y. Wen, Y.H. Yu, Mechanics of fluidization, Chem. Eng. Prog. Symp. Series, 62 (1966) 100-111.

[21] N-Q. Nguyen, A.J.C. Ladd, Lubrication corrections for lattice-Boltzmann simulations of particle suspensions, Phys Rev E. 66 (2002) 046708.

[22] Y. Yamamoto, M. Potthoff, T. Tanaka, T. Kajishima, Y. Tsuji, Large-eddy simulation of turbulent gasparticle flow in a vertical channel: effect of considering inter-particle collisions, J. Fluid Mech. 442 (2001) 303-334.

[23] K.D. Squires, J.K. Eaton, Particle response and turbulence modification in isotropic turbulence, Phys. Fluids 2 (1990) 1191-1203. 
Figures

Figure 1. Flow geometry and coordinate system. Left: top view; right: side view. The pitched-blade turbine pumps liquid in the downward direction.
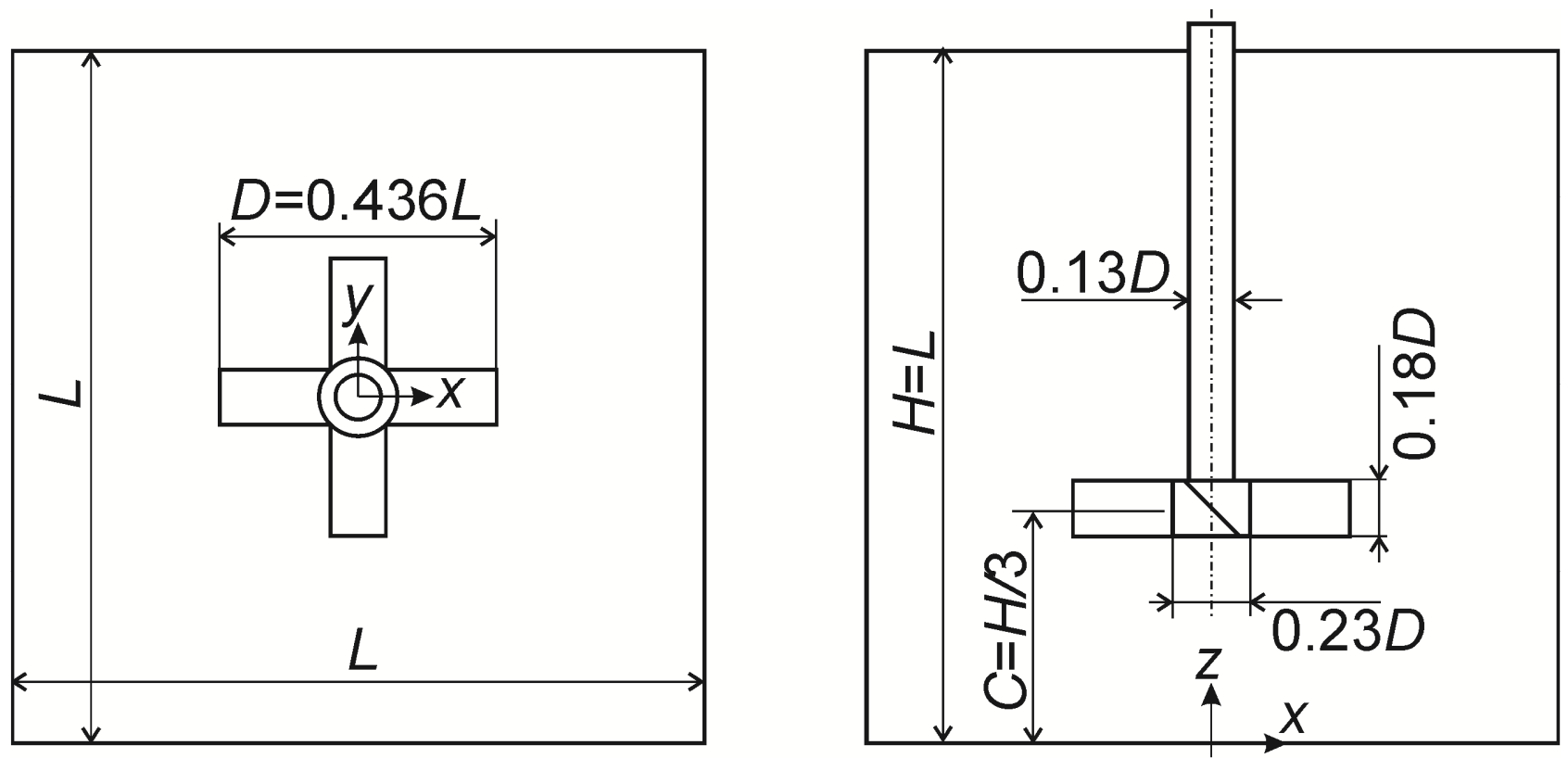
Figure 2. Left: moment of collision between particle $a$ and $b$. Right: particle $a$ breaks in two particles $a_{1}$ and $a_{2}$ that are placed as shown oriented with a random angle $\theta$ around the line connecting the centers of the original particles $a$ and $b$.

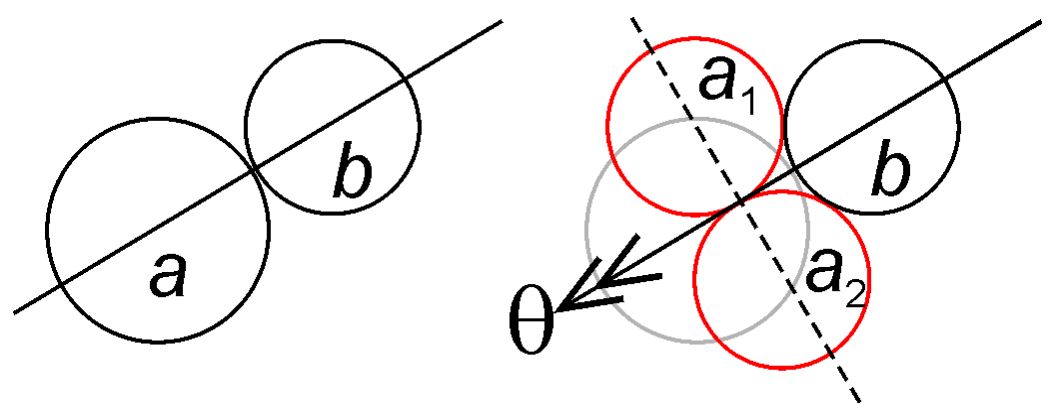


Figure 3. Impressions of particle locations and velocity field in the mixing tank at different moments in time. The contours in the mid plane represent the liquid velocity magnitude. Particle color coding: blue $d=d_{0}$, red $d=2^{-1 / 3} d_{0}$, yellow $d=2^{-2 / 3} d_{0}$, white $d=2^{-1} d_{0}$, black $d=2^{-4 / 3} d_{0}$. The top two panels contain all particles; the bottom four only show particles in a center layer with thickness $0.1 L$. Base-case conditions with particle strength such that $u_{c} / \mathrm{v}_{\text {tip }}=0.2$.
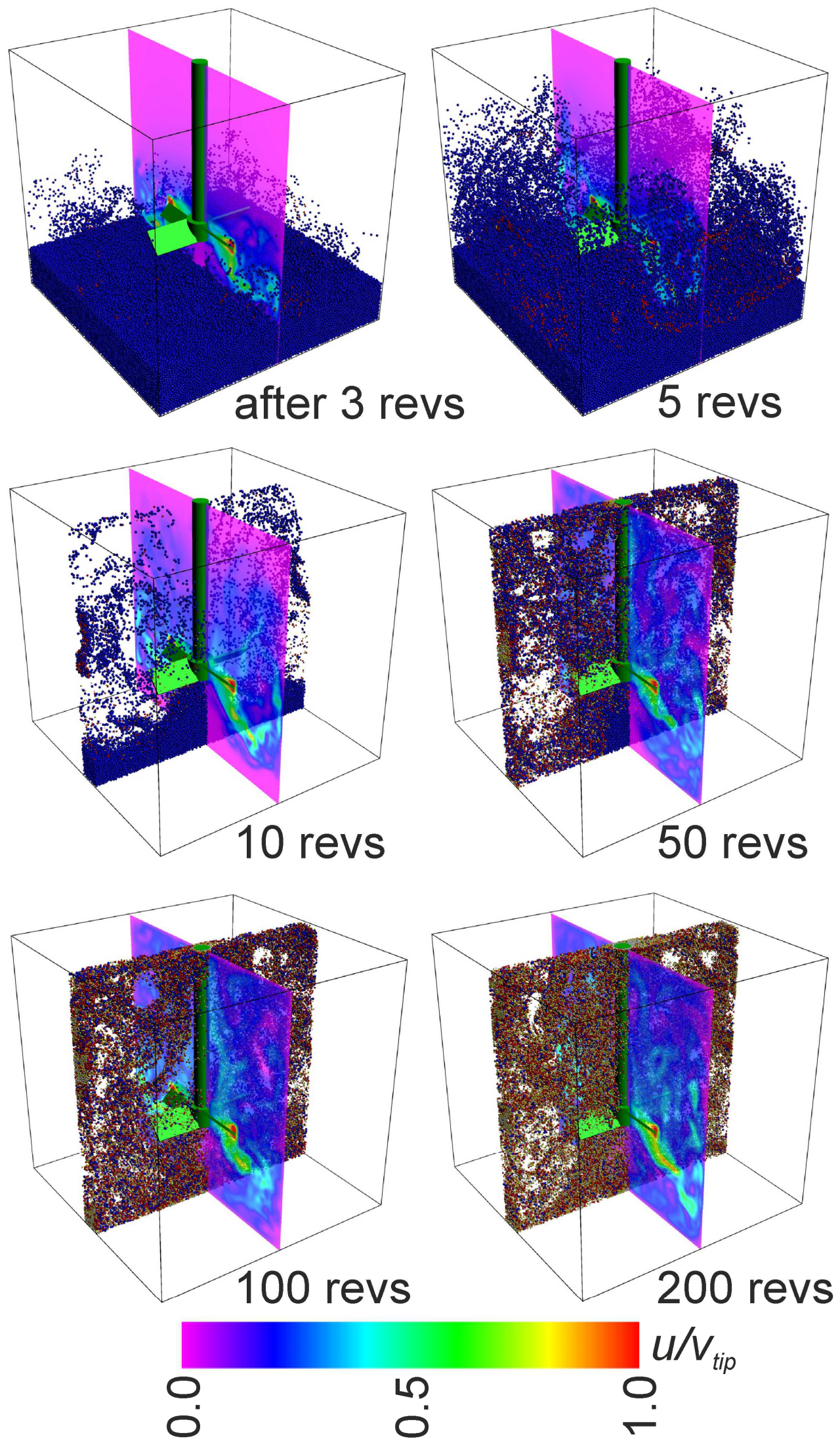
Figure 4. Solids volume fraction distribution in the vertical center plane. Averages over 5 revolutions around (from left to right) moments $t N=10,50,100$, and 200.
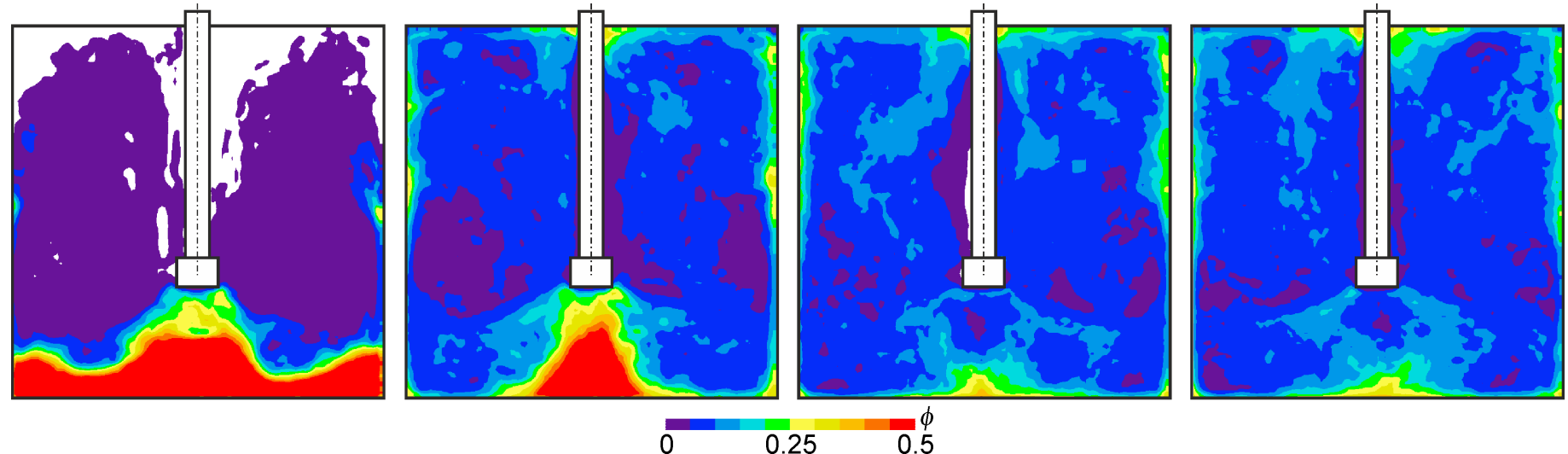

0.5 
Figure 5. A view of particles close to the impeller. This is a detail from the panel at 100 impeller revolutions in Figure 3. Same particle color coding as in Figure 3.

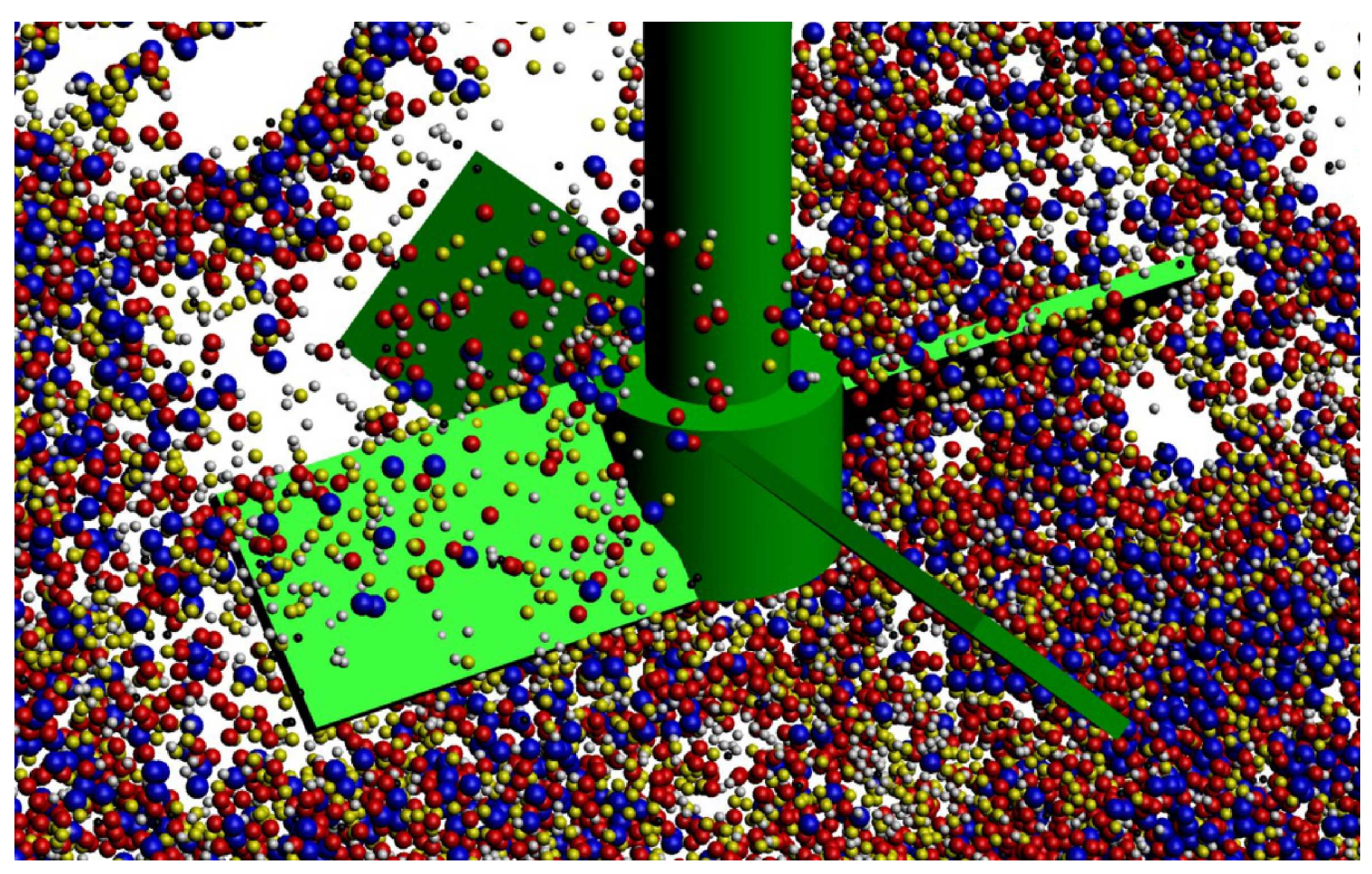


Figure 6. Time series of the number of particles in the tank (relative to the initial number) $n / n_{0}$ (top) and Sauter mean diameter (relative to the initial diameter) $d_{32} / d_{0}$ (bottom) for particles of different strength as indicated. Base-case conditions.

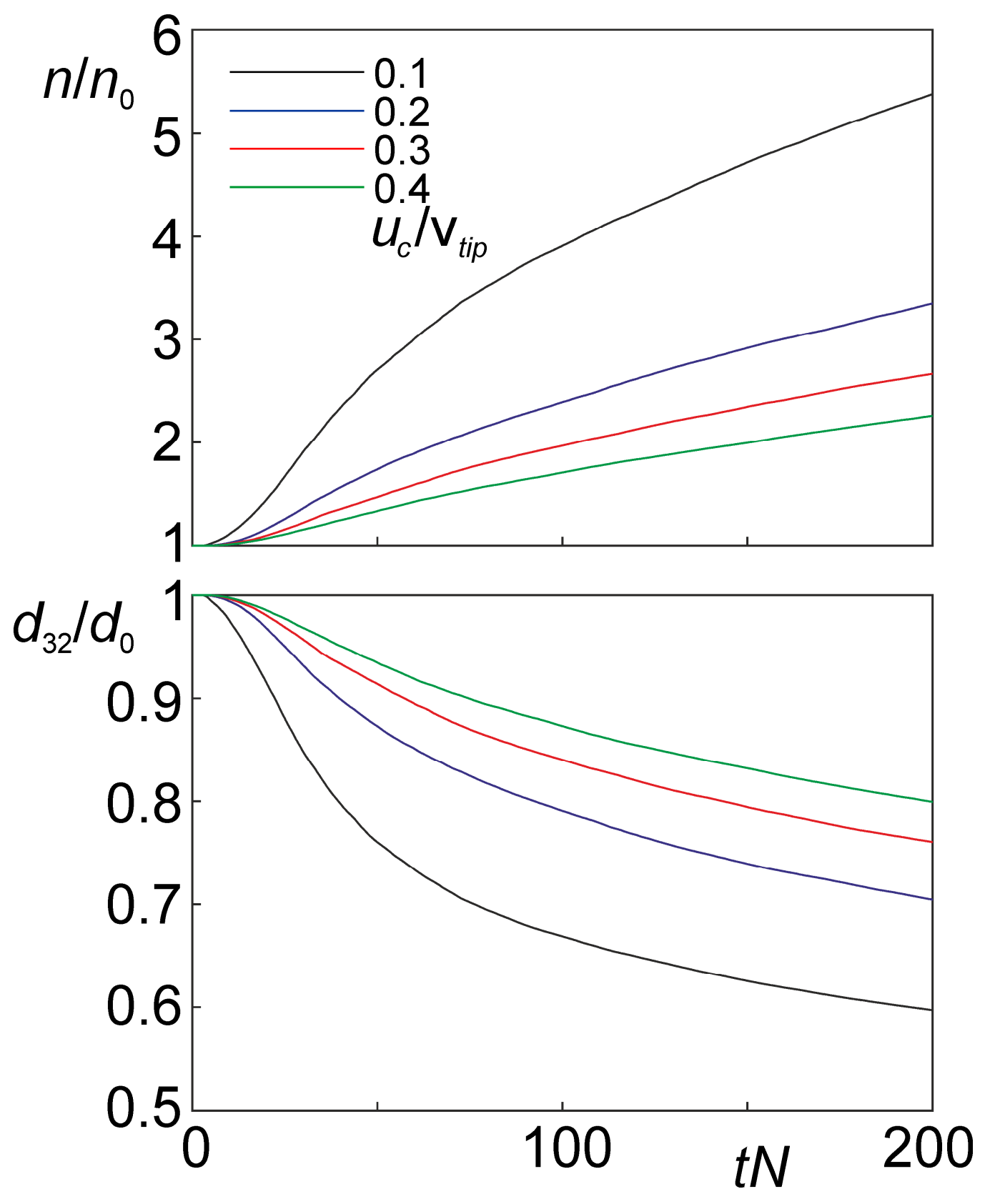


Figure 7. Time series of the number of breakage events per impeller revolution $n_{b}$ (divided by $n_{0}$ ). Particle strength $u_{c} / \mathrm{v}_{\text {tip }}$ as indicated. Breakage type as specified: $\mathrm{p}$-p breakage due particle-particle collision, $\mathrm{p}-\mathrm{i}$ particle-impeller collision, $\mathrm{p}$-w particle-wall collision, and all is the total number.
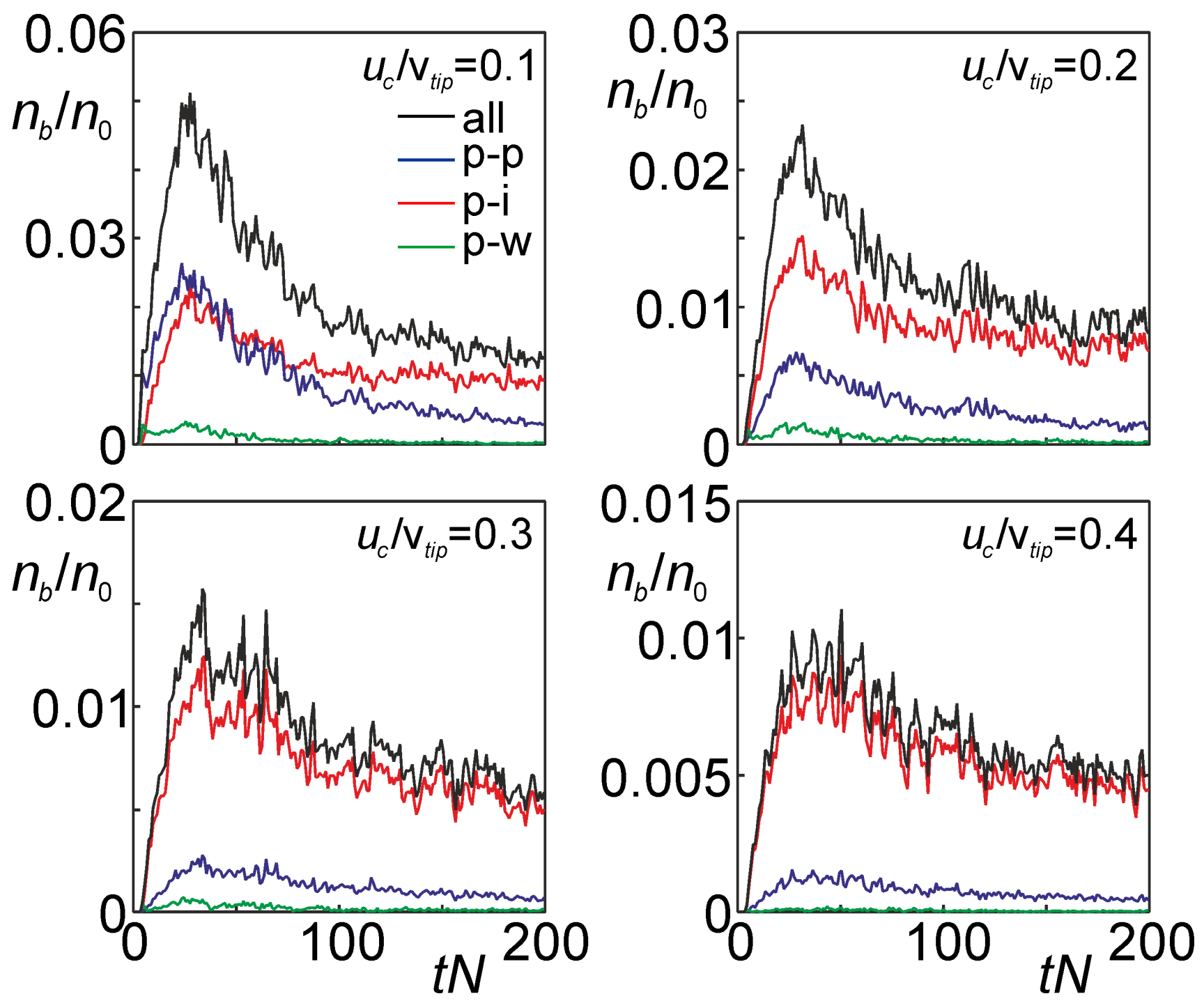
Figure 8. Distribution of the number of breakage events per unit volume and per impeller revolution $n_{b v}$ normalized by the initial number concentration of particles $n_{0} / V$ in the center vertical mid-plane of the tank. Data averaged over 150 impeller revolutions with averaging starting 50 revolution after start up. Left: breakage due to particle-particle collisions; right due to particle impeller collisions. Base-case conditions with $u_{c} / \mathrm{v}_{\text {tip }}=0.2$.
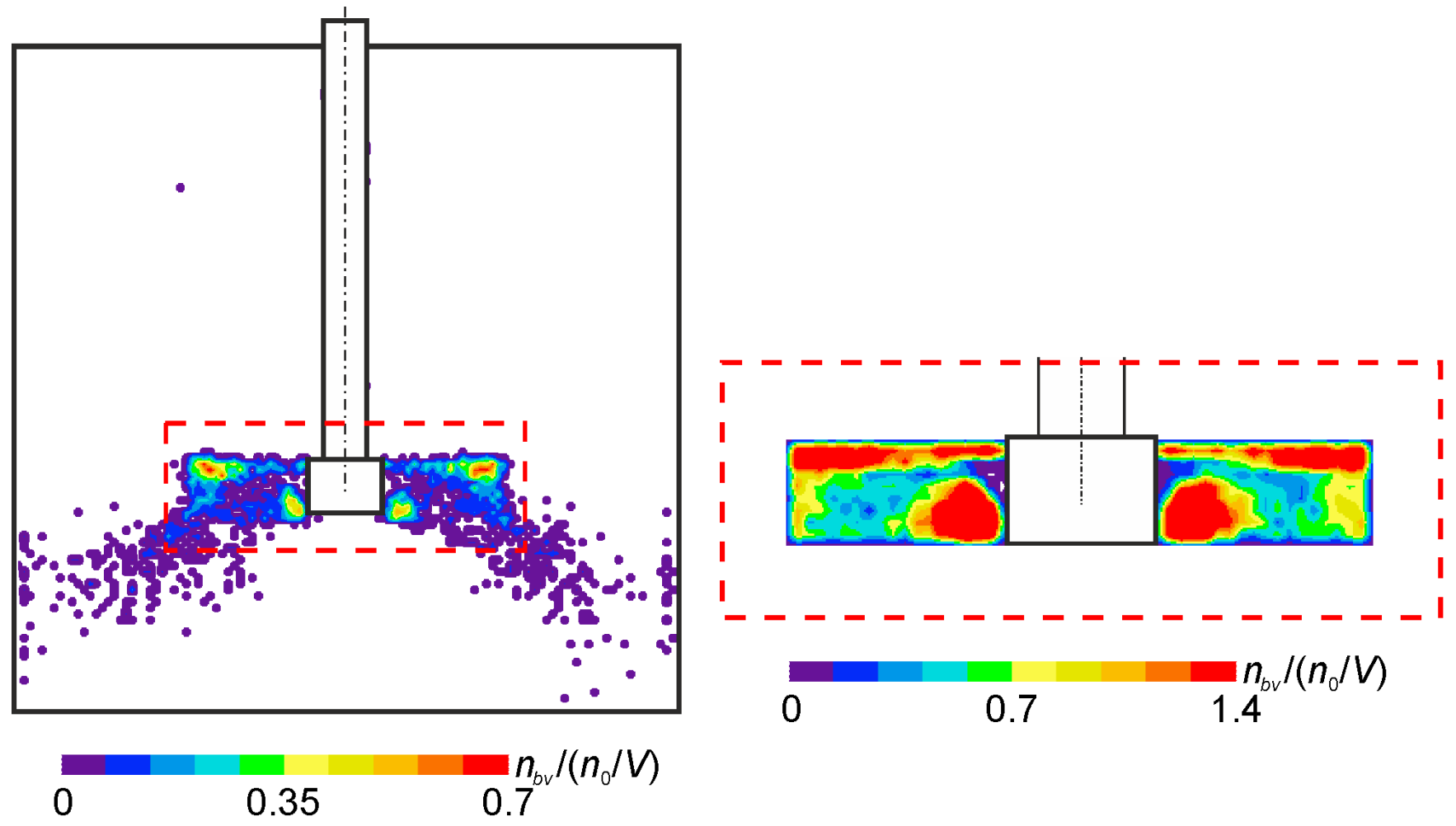

$n_{b v} /\left(n_{0} / V\right)$ 
Figure 9. Instantaneous liquid velocity vector fields in the vertical center plane with an impeller blade passing through. From left to right the blade centerline is $18^{\circ}$ behind the center plane, in the center plane, and $18^{\circ}$ in front of the center plane. The dashed green circle in the left panel highlights the corner vortex in front of the blade.
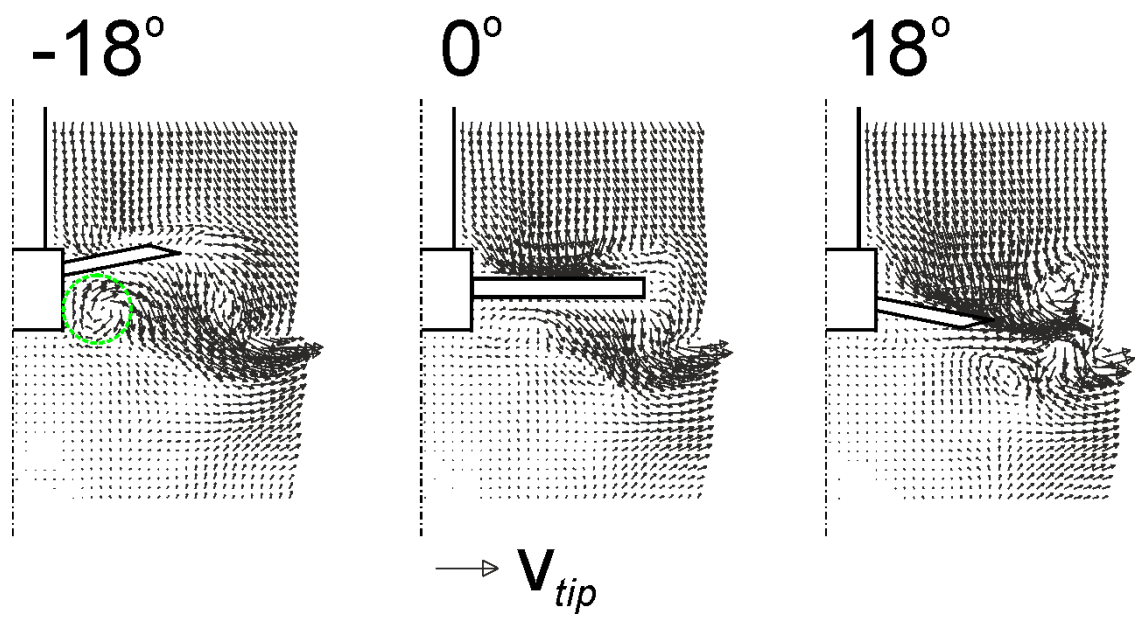
Figure 10. Time average flow characteristics in the center mid-plane. From left to right: velocity magnitude $|\mathbf{u}|$, kinetic energy $k$ contained in the fluctuations, and energy dissipation rate $\varepsilon$; all scaled as indicated. Averaging time 14 impeller revolutions; base case with $u_{c} / \mathrm{v}_{\text {tip }}=0.2$.
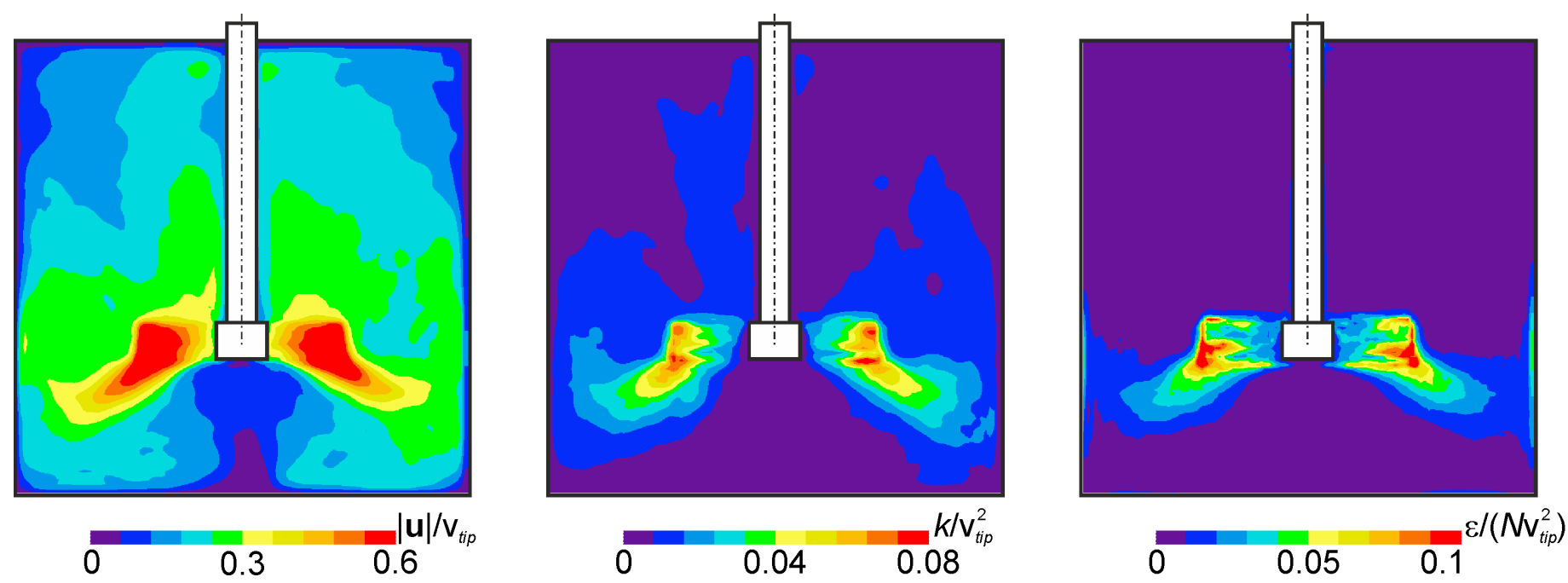
Figure 11. Particle size distribution by volume $\left(V_{d} / V_{p t}\right.$ is the solids volume contained in particles with diameter $d$ over the total solids volume). Evolution in time and effects of solids strength as indicated.

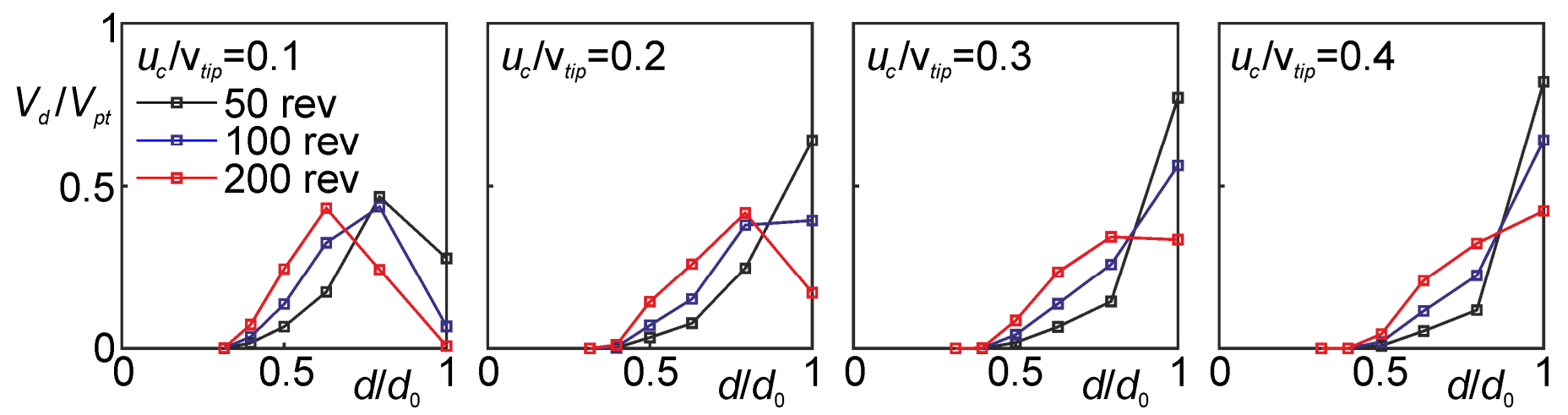

